\title{
Quantitative characterization of absorber and phase defects on EUV reticles using coherent diffraction imaging
}

\author{
Iacopo Mochi, ${ }^{\text {a, } * \text { Sara Fernandez, }},{ }^{\mathrm{a}}$ Ricarda Nebling, ${ }^{\mathrm{a}}$ Uldis Locans, ${ }^{\mathrm{a}}$

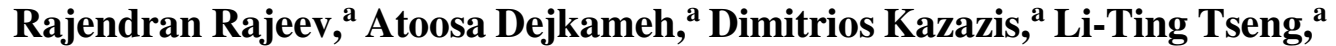 \\ Serhiy Danylyuk, ${ }^{b}$ Larissa Juschkin, ${ }^{\text {,d,e }}$ and Yasin Ekinci ${ }^{\mathrm{a}}$

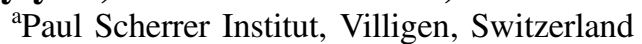 \\ ${ }^{\mathrm{b}}$ Fraunhofer Institute for Laser Technology, Aachen, Germany \\ ${ }^{c}$ Peter Grünberg Institute 9, Semiconductor Nanoelectronics, Research Center Jülich, \\ Jülich, Germany \\ ${ }^{\mathrm{d}}$ RWTH Aachen University, Chair for the Experimental Physics of Extreme Ultraviolet EUV, \\ Aachen, Germany \\ ${ }^{\mathrm{e} J u ̈ l i c h}$ Aachen Research Alliance for Fundamentals of Future Information Technology, \\ Aachen, Germany
}

\begin{abstract}
Background: Reliable photomask metrology is required to reduce the risk of yield loss in the semiconductor manufacturing process as well as for the research on absorber materials. Actinic pattern inspection (API) of EUV reticles is a challenging problem to tackle with a conventional approach. For this reason, we developed RESCAN, an API platform based on coherent diffraction imaging.
\end{abstract}

Aim: We want to verify the sensitivity of our platform to absorber and phase defects.

Approach: We designed and manufactured two EUV mask samples with absorber and phase defects, and we inspected them with RESCAN in die-to-database mode.

Results: We reconstructed an image of an array of programmed absorber defects, and we created a defect map of our sample. We inspected two programmed phase defect samples with buried structures of 3.5 and $7.8 \mathrm{~nm}$ height.

Conclusions: We verified that RESCAN, in its current configuration, can detect absorber defects in random patterns and buried (phase) defects down to $50 \times 50 \mathrm{~nm}^{2}$.

(C) 2020 Society of Photo-Optical Instrumentation Engineers (SPIE) [DOI: 10.1117/1.JMM.19.1.014002]

Keywords: EUV; actinic pattern inspection; phase defects; pellicle; lensless microscopy.

Paper 19096 received Nov. 17, 2019; accepted for publication Jan. 16, 2020; published online Jan. 30, 2020.

\section{Introduction}

RESCAN is a reflective-mode EUV mask scanning lensless imaging microscope currently under development at the Paul Scherrer Institute, with the goal to enable defect inspection on EUV reticles for current and future lithography nodes. ${ }^{1,2}$ This novel microscope can operate in die-todie or die-to-database mode by comparing the images of subsequent regions of the reticle with a reference and detecting the defects by evaluating differences in the signals. ${ }^{3}$ RESCAN is based on coherent diffraction imaging (CDI). For this reason, it does not need complex and expensive EUV optics, and it is capable of measuring the phase and the magnitude of the complex reflection function of the sample. The absence of optical elements in close proximity to the sample makes it

*Address all correspondence to Iacopo Mochi, E-mail: iacopo.mochi@psi.ch

$1932-5150 / 2020 / \$ 28.00$ C 2020 SPIE 


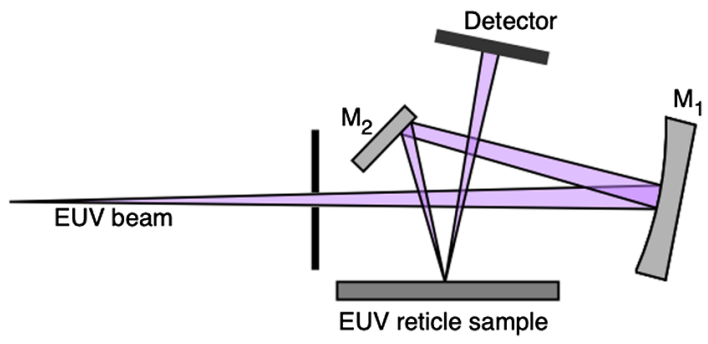

Fig. 1 Schematic optical layout of RESCAN. The EUV beam comes from a dedicated beamline at the Swiss Light Source. The light is focused by a multilayer-coated toroidal mirror (M1) and folded onto the sample by a flat mirror (M2). The beam incidence angle on the EUV reticle sample is 6 deg.

possible to inspect reticles protected by EUV pellicles, ${ }^{4,5}$ which is a requirement for any mask inspection tool integrated in the lithography process.

\subsection{RESCAN Working Principle and Layout}

RESCAN is a lensless actinic microscope dedicated to EUV mask research, installed at the Swiss Light Source. The microscope has a dedicated beamline equipped with a tunable undulator and a monochromator with a bandwidth $\lambda / \Delta \lambda=1500$. The optical layout of the tool is shown in Fig. 1. The beam entering the chamber has a wavelength $\lambda$ of $13.5 \mathrm{~nm}$, a numerical aperture $\mathrm{NA}_{0} \simeq 5 \cdot 10^{-4}$, and is clipped by a selectable circular aperture with a diameter ranging from 0.2 to $4 \mathrm{~mm}$. The beam is then focused onto the sample by a multilayer-coated toroidal mirror with a focal length of $120 \mathrm{~mm}$. The angle of incidence of the beam on the sample is $6 \mathrm{deg}$. The illumination NA value ranges between $1.7 \cdot 10^{-2}$ and $1.7 \cdot 10^{-3}$. The beam reflected and diffracted by the sample is collected by a $2048 \times 2048$ pixel CCD (Princeton Instruments MTE-2) with a pixel size of $13.5 \mu \mathrm{m}$ at a distance of $62 \mathrm{~mm}$ from the sample.

Our imaging approach is based on ptychography, ${ }^{6-8}$ a CDI technique that consists of scanning a sample with a coherent probe allowing some degree of overlap between the illuminated regions. The diffraction patterns are recorded with a pixel detector. As in other CDI approaches, when the diffraction pattern is recorded, the phase information is lost and it must be retrieved to reconstruct the sample image. In ptychography, the phase is obtained from the information redundancy generated by the overlap of the illuminated regions.

Defect detection in RESCAN is performed comparing the reconstructed image to a reference one obtained from a control region (die-to-die) or calculated from the mask design (die-todatabase) properly aligned and scaled to match the inspected area. A threshold is applied to the difference between the two images to avoid false positives that may arise from artifacts in the reconstruction or from approximations in the reference aerial image calculation. We have demonstrated that RESCAN is capable of detecting programmed defects on EUV logic patterns using die-to-die and die-to-database approaches, ${ }^{3}$ and we tested it on pellicle-covered samples, evaluating the impact of different types of pellicles on the image quality. ${ }^{9}$ We describe here the experiments performed in order to evaluate the inspection sensitivity of RESCAN for absorber and phase defects.

\section{Absorber Defects Inspection}

The theoretical imaging resolution $r$ of RESCAN in the configuration described in the previous section is given as

$$
r=0.61 \frac{\lambda}{\mathrm{NA}}=0.61 \frac{13.5 \mathrm{~nm} \cdot 62 \mathrm{~mm}}{1024 \cdot 13.5 \mu \mathrm{m}}=37 \mathrm{~nm} .
$$

To verify the sensitivity of RESCAN to absorber defects, we designed a sample layout with random structures with a critical dimension (CD) of $200 \mathrm{~nm}$. The sample design includes a 
(a)

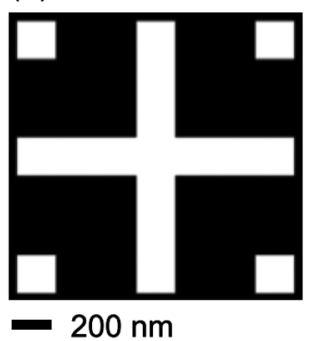

(b)

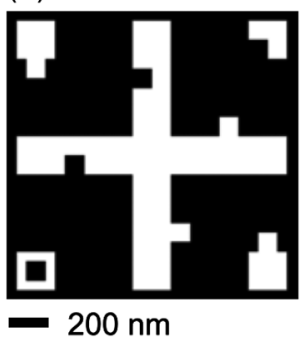

(c) $200 \mathrm{~nm}$
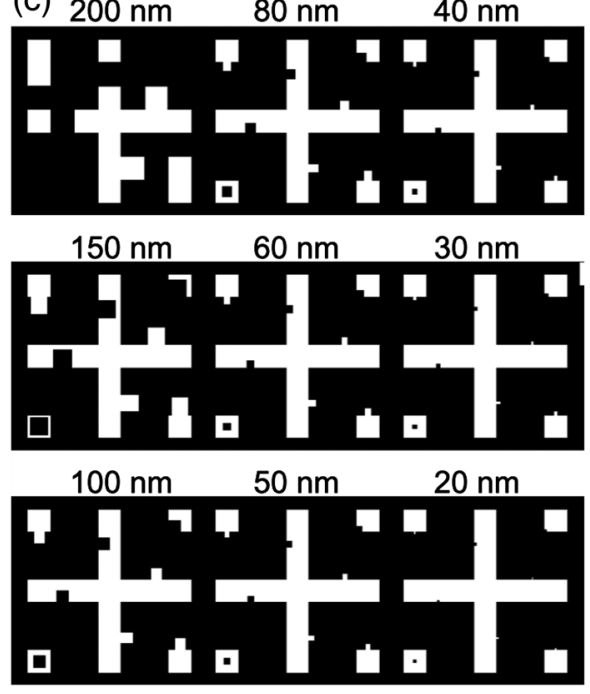

- $400 \mathrm{~nm}$

Fig. 2 (a) Control structure. (b) Defect arrangement on the control structure. (c) Matrix layout of the defective control structure with defect sizes from 20 to $200 \mathrm{~nm}$. This matrix is embedded in a $26 \times 26 \mu \mathrm{m}$ random pattern area with $200-\mathrm{nm}$ CD. Note that, to print the defects to size, the exposure dose was locally adjusted to compensate for the linearity error of the E-beam writer.

control structure and a copy of it with programmed intrusion, extrusion, and pin-dot defects as shown in Fig. 2. The programmed defect structure is replicated in a $3 \times 3$ array with defect sizes from 200 to $20 \mathrm{~nm}$. The layout was printed using e-beam lithography (EBL) on a multilayercoated silicon wafer with a 140-nm thick hydrogen silsesquioxane (HSQ) absorber layer. The choice of HSQ as absorber was motivated by the availability of a stable and reliable in-house lithography process for this material as opposed to other, better performing, absorber stacks such as TaBN or nickel alloys. ${ }^{10}$ The quality of the pattern was verified with scanning electron microscopy (SEM), confirming that even the smallest programmed defects were printed to size.

We inspected the sample with RESCAN over a $30 \times 30 \mu \mathrm{m}^{2}$ area centered on the array of programmed defect structures. In this experiment, we illuminated the sample with $\sim 2 \cdot 10^{9}$ EUV photons $/ \mu \mathrm{m}^{2}$. The reconstructed image containing the defect array is shown in Fig. 3(a).

(a)
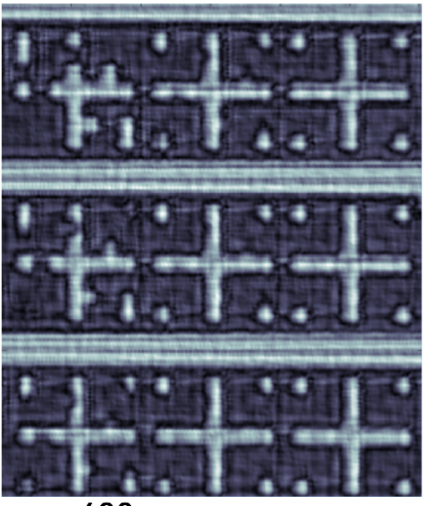

(b)

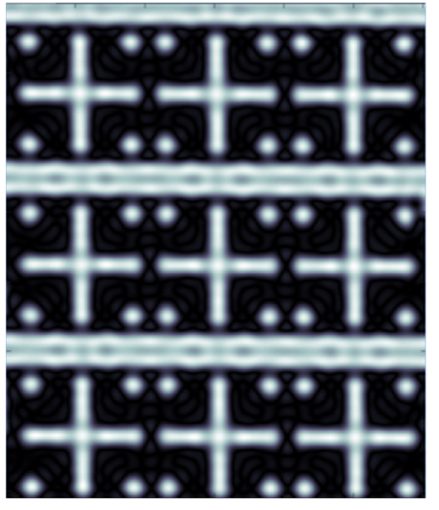

(c)

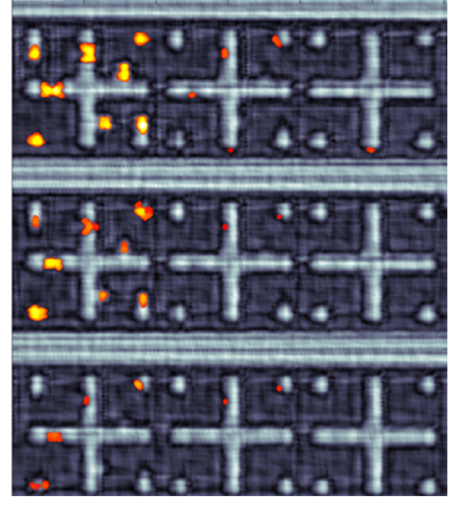

Fig. 3 (a) Detail of the sample image reconstructed with RESCAN showing the matrix of control structures with programmed defects. (b) Aerial image calculated from a version of the sample layout with defect-free control structures. (c) The defect map calculated as the absolute value of the difference between the intensities of $(a)$ and $(b)$ is shown in color, superimposed to the reconstructed image (a). 


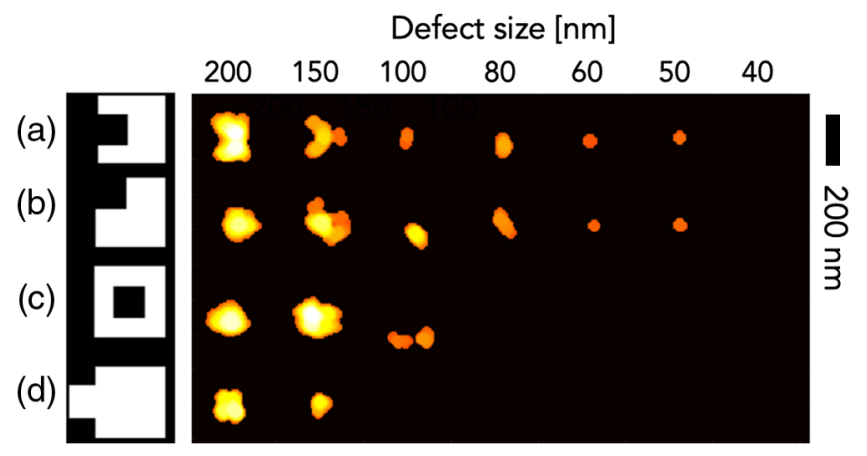

Fig. 4 The single images of the defects shown in the map of Fig. 3(c) are arranged here in an array as a function of defect type and size. (a) Line intrusion defects, detected down to a size of $50 \mathrm{~nm}$. (b) Corner intrusion defects, detected down to a size of $50 \mathrm{~nm}$. (c) Pin-dot defects, detected down to a size of $100 \mathrm{~nm}$. (d) Line extrusion defects, detected down to a size of $150 \mathrm{~nm}$.

We calculated the reference aerial image shown in Fig. 3(b), starting from a mask design version without programmed defects. Assuming plane wave illumination, we propagated the mask through a perfect lens with pupil shape and NA matching the ones defined by the RESCAN detector and a magnification of one. The intensity of the simulated aerial image was then scaled to match the one of the reconstructed image. The absolute value of the difference of the two images constitutes the sample's defect map. To limit the presence of false positives, a threshold was applied to the defect map. False-positive signals are mainly caused by the artifacts in the image reconstruction process and by the oversimplified model used to calculate the reference image that does not take into consideration the 3-D structure of the sample, the angle of incidence of the illumination, and the optical properties of the absorber and the multilayer. The effect of artifacts in the image reconstruction process can be observed comparing Figs. 3(a) and 3(b). The dark region of the reconstructed image is not as uniform as the corresponding region of the simulated aerial image. Let $N$ be the standard deviation of the intensity in the dark region of Fig. 3(a), and let $S$ be the average intensity of the bright region of the same image. The signal-tonoise ratio of the reconstructed image is given by $S / N$ and it is equal to 5.4. In this case, to avoid the detection of false positives, we choose a threshold of 50\% on the defect map intensity. The thresholded defect map is shown in color, superimposed to the reconstructed image in Fig. 3(c).

The single images of the detected defects are shown in Fig. 4, where it can be observed that line intrusions (a) and corner intrusions (b) are detected down to a size of $50 \mathrm{~nm}$, while pin-dot defects (c) and line extrusions (d) are detected down to 100 and $150 \mathrm{~nm}$ size, respectively.

\section{Phase Defect Inspection}

One of the advantages of CDI compared to standard imaging techniques is that from the complex amplitude of the reconstructed object, it is possible to measure its phase as well as its magnitude. This is of particular interest in the context of EUV mask inspection, where defects buried under or within the multilayer can create significant artifacts in the aerial images. These defects, often undetectable with SEM, atomic force microscopy (AFM), or deep ultraviolet imaging, ${ }^{11}$ can be investigated effectively with RESCAN. To evaluate the sensitivity of the microscope to phase defects, we created a sample with programmed structures buried under the multilayer. The buried structures were defined by EBL on a silicon wafer spin-coated with PMMA resist. Then, a thin layer of carbon was deposited and the patterns were revealed by a lift-off process. We repeated this process on two samples with different carbon thickness ( 7.8 and $3.5 \mathrm{~nm}$ ), and we verified the resulting structures profile with AFM as shown in Fig. 5. The AFM scan confirmed that the thickness values of the carbon layer were correct, but also showed that the lift-off process failed to remove some carbon on the edges of the structures. This effect can be prevented by increasing the thickness of the PMMA resist or using a bilayer resist in order to facilitate the lift-off process. 


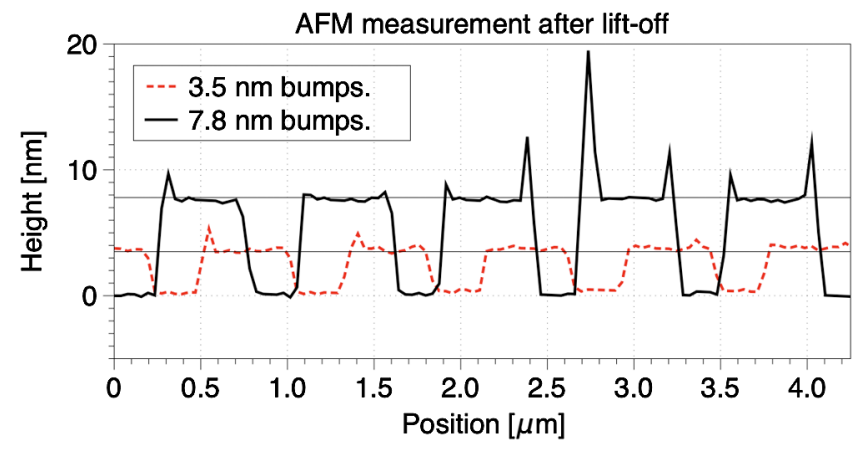

Fig. 5 AFM profile scan of $500 \mathrm{~nm}$ wide carbon structures on two different samples. The profile scan confirms that the thickness values of the carbon structures are 3.5 and $7.8 \mathrm{~nm}$. It also shows that the lift-off process failed to remove some carbon at the edges of the structures.

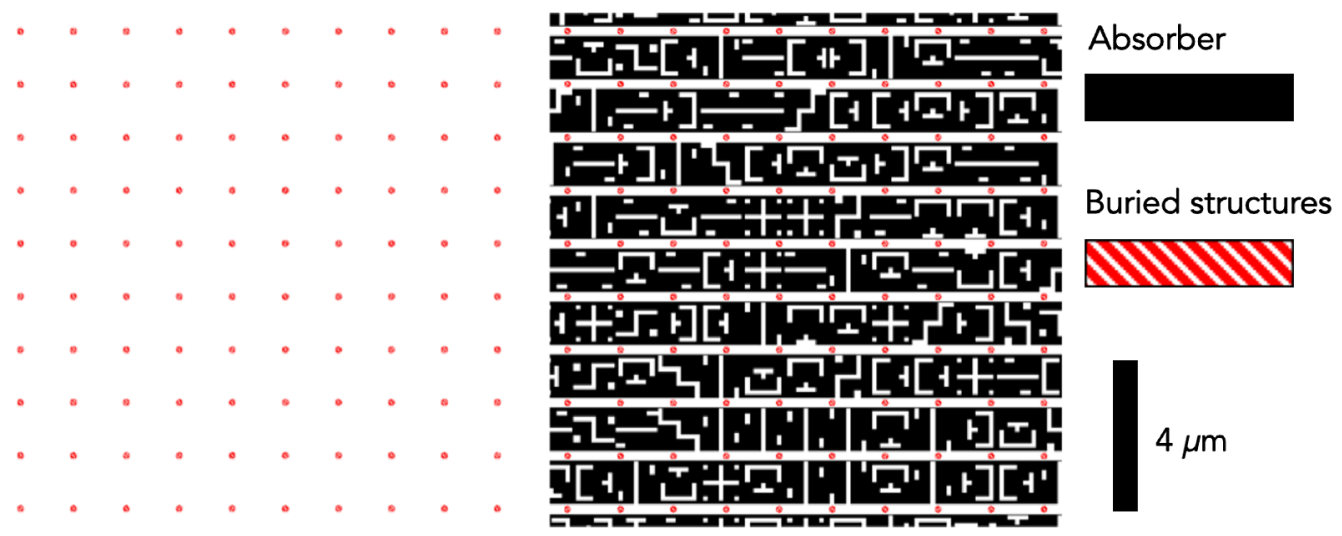

Fig. 6 Detail of the sample layout including buried carbon structures and HSQ absorber pattern. The carbon structures consist of $800 \mathrm{~nm} \mathrm{1:1} \mathrm{lines} \mathrm{and} \mathrm{dots} \mathrm{with} 200 \mathrm{~nm}$ diameter and $2 \mu \mathrm{m}$ pitch. The absorber pattern consists of a logic-like pattern with 200-nm CD.

The samples were then coated with a MoSi multilayer with 40 bilayers, resulting in a $64 \%$ EUV reflectance at 6-deg incidence angle.

The samples were finally coated with a 140-nm HSQ layer and patterned with EBL. The resulting sample layout is shown in Fig. 6, where the absorber pattern is represented in black and the buried structures are stippled with red lines. Among the carbon structures we generated, we included an array of 200-nm diameter dots with a pitch of $2 \mu \mathrm{m}$, and we aligned a random logic-like absorber pattern on top of it. The absorber pattern has a CD of $200 \mathrm{~nm}$ and horizontal trenches (power rails) with a width of $400 \mathrm{~nm}$. The trenches were aligned to the carbon dots in order to have phase structures on a clear region of the sample.

A detail of this arrangement is shown in Fig. 7, where we also included an SEM micrograph of the same region. In the SEM image, the contours of the defects are visible because of the extra carbon left by the lift-off process, but the buried structures are otherwise undetected.

We inspected the sample with 3.5-nm-thick carbon structures in RESCAN, and we extracted the phase and the magnitude from the measured complex amplitude. The contour of the buried defects is clearly visible in the magnitude image [Fig. 8(a)], but, more interestingly, the defects phase signature is also detected with good contrast in the phase map [Fig. 8(b)]. The average phase difference between the defect-free areas and carbon bump peaks is $\overline{\Delta \phi}=(0.93 \pm 0.05) \pi$. We tested the 7.8-nm high structures on an absorber-free region with an array of phase contacts with $50 \mathrm{~nm}$ diameter and $2 \mu \mathrm{m}$ pitch. The reconstructed magnitude and phase maps are shown in Figs. 9(a) and 9(b), respectively. Even in this case, the defects are visible in the magnitude map, but their phase contrast is considerably lower than in the previous case since the defect height is close to half of the wavelength and should produce a phase shift of roughly $2 \pi$. The average phase difference measured on the defect peaks (modulus $2 \pi$ ) is actually $\overline{\Delta \phi}=(0.13 \pm 0.05) \pi$. 
(a)

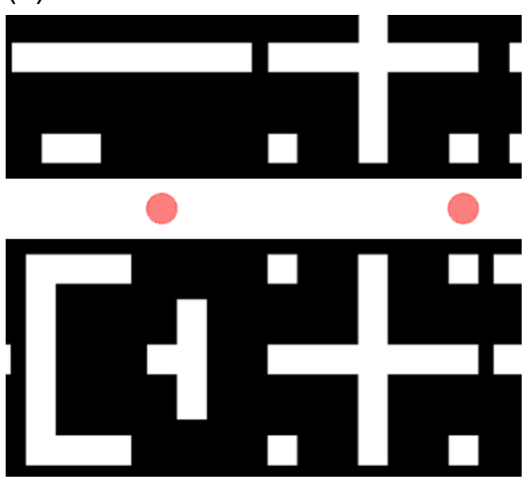

(b)

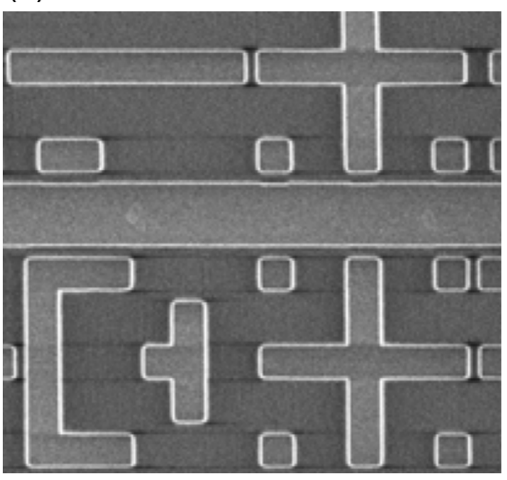

$1.2 \mu \mathrm{m}$

Fig. 7 Detail of the random logic-like pattern shown in Fig. 6. (a) The 200-nm carbon dots have been aligned to the horizontal 400-nm power rails in the pattern. (b) SEM micrograph of the region shown in (a) with 7.8-nm carbon structures. The edges of the buried structures are barely visible because of the extra carbon left by the lift-off process. The central part of the dots was not detected by the SEM.

(a)

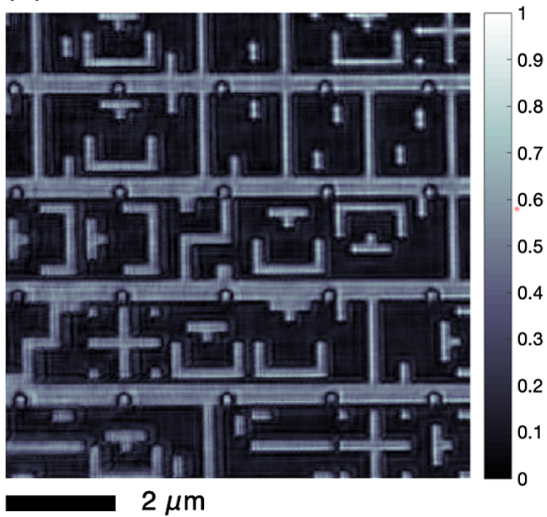

(b)

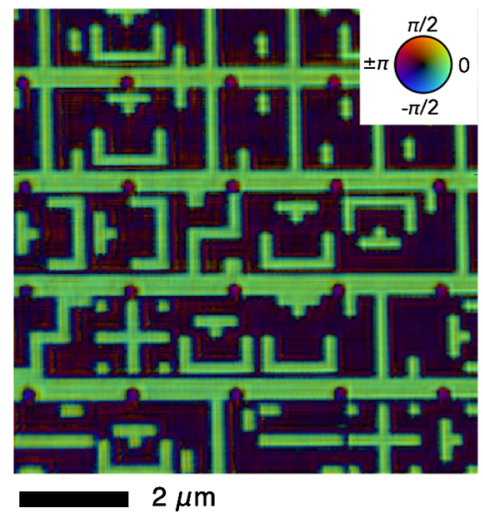

Fig. 8 Detail of the reconstructed image of the sample with 3.5-nm high carbon structures. (a) Reconstructed magnitude map. (b) Reconstructed phase map.

(a)

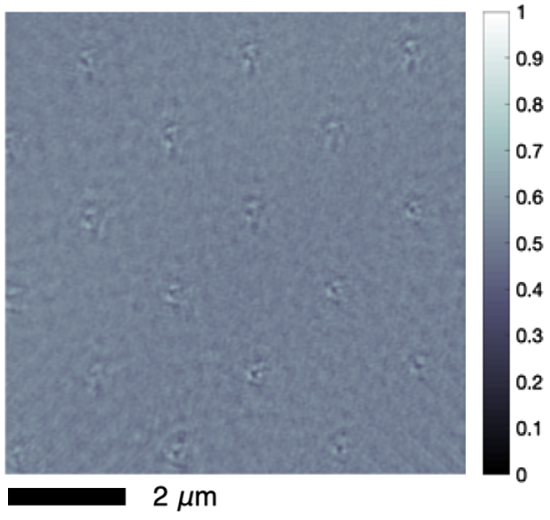

(b)

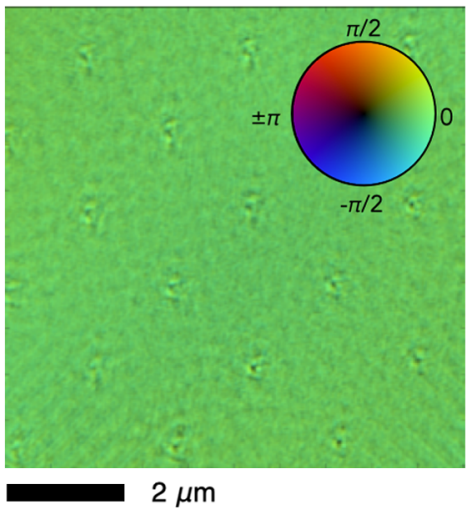

Fig. 9 Detail of the reconstructed image of the sample with 7.8-nm high carbon structures. This area of the sample is absorber-free and under the multilayer there is an array of carbon dots with a diameter of $50 \mathrm{~nm}$ and a pitch of $2 \mu \mathrm{m}$. (a) Reconstructed magnitude map. (b) Reconstructed phase map. 
To assess the accuracy of the phase reconstruction, we needed to compare the phase values obtained with RESCAN to a reference. The phase variation induced by a surface bump on an ideal flat mirror can be calculated as

$$
\Delta \phi=\frac{4 h \pi}{\lambda \cos \theta},
$$

where $h$ is the bump height, $\lambda$ is the wavelength, and $\theta$ is the illumination angle of incidence. In the present case though, it is necessary to take into account the smoothing effect that the multilayer deposition has on the defect topography and the penetration depth of the EUV light inside the multilayer.

We used an empirically calibrated model to predict the deformation of each $\mathrm{Mo} / \mathrm{Si}$ bi-layer in the presence of the carbon dots on our programmed defect mask. The calculations of bilayers deformation from a defined defect are based on the nonlinear continuum model ${ }^{12,13}$ with nonzero coefficients correlated to condensation/evaporation (sputter/redeposition), surface diffusion, and contraction due to silicide interlayers formation, that describe well the standard Optixfab multilayer fabrication process. These coefficients were derived from experimental studies ${ }^{14}$ by comparison of TEM images of lamella cuts out of programmed buried defects with the modeled multilayer deformation. The resulting vertical displacement $h$ of each bilayer in the presence of the carbon dots on our programmed defect mask is shown in Fig. 10. The vertical displacement was calculated as the height difference between a point corresponding to the center of the defect and a point $100 \mathrm{~nm}$ away. The value of $h$ as a function of the layer number, for the case of a 7.8-nm-high defect, is shown in Fig. 11. We calculated the reflectance contribution $w$ of each bilayer by estimating the total reflectance $R(n)$ of a standard EUV multilayer stack with $n$ bilayers with $n$ from 1 to 40 and normalizing its difference

$$
w(n)=\frac{R(n)-R(n-1)}{R(40)},
$$

where $R(n)$ was calculated using a publicly available MATLAB ${ }^{\circledR}$ function ${ }^{15}$ assuming an incidence angle of $6 \mathrm{deg}$, a wavelength of $13.5 \mathrm{~nm}$, and a multilayer stack described by the values in Table 1 . The effective defect height is obtained weighting the height of each bilayer with its relative reflectance, so that Eq. (2) becomes

$$
\Delta \phi=\sum_{m=1}^{40} h(m) w(41-m) \frac{4 \pi}{\lambda \cos \theta}
$$

(a)

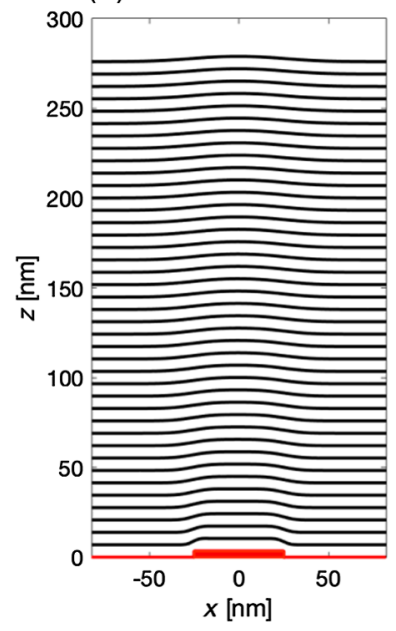

(b)

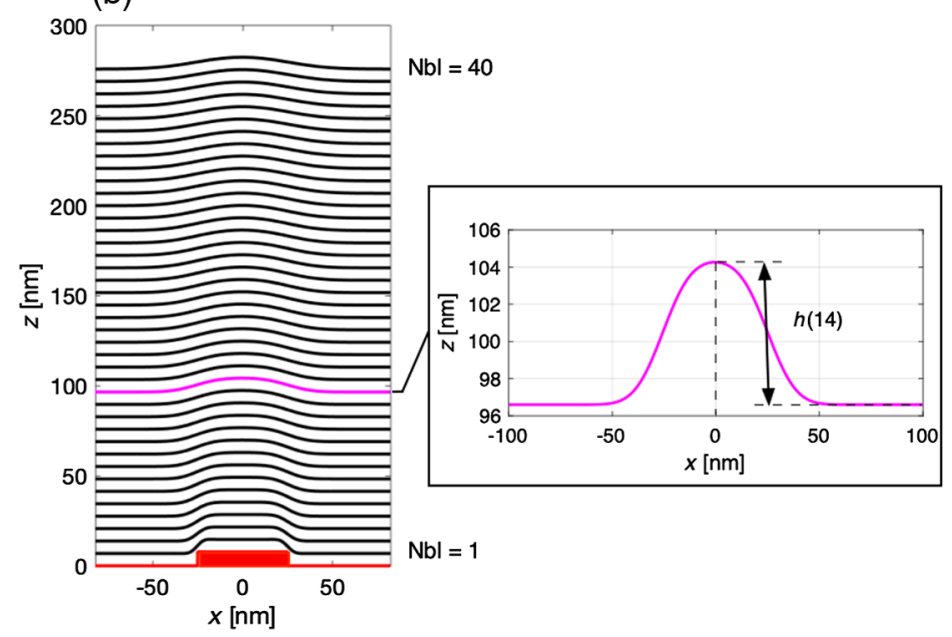

Fig. 10 Simulation of the multilayer deformation induced by (a) a $50 \times 50 \mathrm{~nm}$ carbon dot with $3.5 \mathrm{~nm}$ height and (b) a $50 \times 50 \mathrm{~nm}$ carbon dot with $7.8 \mathrm{~nm}$ height. The detail of (b) shows the peak deformation for the 14-th bilayer. The deformation $h$ is calculated as the difference of the bilayer $z$ positions in correspondence of the center of the carbon dot and $100 \mathrm{~nm}$ away from it. 


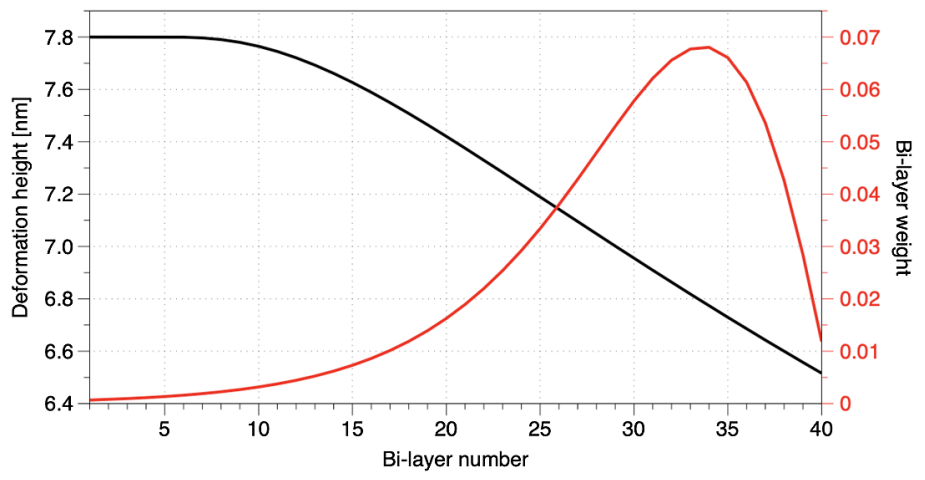

Fig. 11 The black curve represents the deformation height $h$ of the Mo/Si bilayers in the presence of a $50 \times 50 \mathrm{~nm}$ carbon dot with a height of $7.8 \mathrm{~nm}$. The height $h$ is calculated as the difference of the layer $z$ position for an $x$ value corresponding to the center of the defect and the layer $z$ position $100 \mathrm{~nm}$ away from it. The red curve represents the relative reflectance contribution of each bilayer.

Table 1 The multilayer is composed of MoSi bilayers. At the interface between the layers, an intermixing region of $\mathrm{MoSi}_{2}$ with variable thickness forms. Note that the standard multilayer for EUV masks is capped with a 2-nm Ru layer to improve its resistance to oxidation, ${ }^{16}$ but no capping layer is present on the sample used in these experiments.

\begin{tabular}{lcccc}
\hline \hline Layer & Material & Thickness $(\mathrm{nm})$ & $n$ & $k$ \\
\hline 1 & $\mathrm{Si}$ & 2.506 & 0.99932 & -0.00183 \\
2 & $\mathrm{MoSi}_{2}$ & 0.802 & 0.9693 & -0.00433 \\
3 & $\mathrm{Mo}$ & 1.904 & 0.92108 & -0.00644 \\
4 & $\mathrm{MoSi}_{2}$ & 1.844 & 0.9693 & -0.00433 \\
5 & $\mathrm{Si}$ & $1 \mathrm{e}$ & 0.99932 & -0.00183 \\
\hline \hline
\end{tabular}

Table 2 Comparison of the predicted peak phase generated by the programmed defects and the relative values measured with RESCAN.

\begin{tabular}{lccc}
\hline \hline Defect height $(\mathrm{nm})$ & Effective height $(\mathrm{nm})$ & Predicted phase & Measured phase \\
\hline 7.8 & 6.97 & $0.08 \pi$ & $(0.13 \pm 0.05) \pi$ \\
3.5 & 3.14 & $0.93 \pi$ & $(0.93 \pm 0.05) \pi$ \\
\hline \hline
\end{tabular}

The comparison between the phase of the defects detected with RESCAN and their expected values is reported in Table 2. In both cases, the predicted phase matches the measured within the estimated uncertainty. The discrepancy between the measured and the predicted phase value for the 7.8-nm-high defects might be due to the carbon residue around the edge of the buried structures shown in Fig. 5, but this must be verified on a new sample manufactured with an optimized lift-off process.

\section{Conclusions}

The RESCAN actinic pattern inspection (API) microscope is a research and development platform for EUV mask inspection based on lensless imaging. After demonstrating the feasibility of API in die-to-die and in die-to-database mode, ${ }^{3}$ and the ability of the microscope to inspect samples equipped with EUV pellicles, ${ }^{9}$ we wanted to test the current defect sensitivity of RESCAN for absorber and buried structures. To investigate RESCAN's sensitivity to absorber 
defects, we fabricated a sample consisting of a multilayer-coated silicon wafer and a 140-nm thick HSQ absorber layer. We used EBL to pattern a random logic-like layout with a series of absorber defects of different types and sizes. Inspecting the sample with RESCAN, we generated a defect map that shows line intrusion and corner defects as small as $50 \times 50 \mathrm{~nm}^{2}$.

We also tested the sensitivity of RESCAN to phase defects. For this purpose, we fabricated two test samples containing defects generated by carbon structures buried under the multilayer. We used carbon bumps of 3.5 and $7.8 \mathrm{~nm}$. We used the complex amplitude of the images reconstructed with RESCAN to generate magnitude and phase maps of the samples, and we observed that we can detect phase defects on clear and patterned regions. The smallest defects available on the test sample are 50-nm diameter dots, and we were able to detect them both in the magnitude and in the phase domain. We used an empirically calibrated model to predict the deformation of the multilayer in the presence of the buried defects, and we calculated the effective defect height based on the relative reflectance of each bilayer. Finally, we verified that 3.5- and 7.8-nm buried defects generate phase disturbance that match the values predicted using our model with an uncertainty of $0.05 \pi$.

\section{Acknowledgments}

We wish to acknowledge the contribution of the technical support team at PSI, Michaela Vockenhuber, Markus Kropf, and Josè Gabadinho, and thank OptixFab for providing the multilayer deposition on the test samples. The authors also thank Jan Biller, Ye Wei, and Mikheil Mebonia for the development and calibration of layers deformation modeling tool. Their studies were supported by the ZIM program of German Federal Ministry of Economics and Technology (BMWi), project "Entwicklung von defektfreien Multilayer-Optiken für Hochtechnologie Spezialanwendungen" (KF3417001DF4). L.J. acknowledges financial support from the Helmholtz Association for a Helmholtz Professorship (Initiative and Networking Fund). This project has received funding from the Electronic Component Systems for European Leadership Joint Undertaking under Grant Agreement No. 783247-TAPES3. This Joint Undertaking receives support from the European Union's Horizon 2020 research and innovation program and Netherlands, Belgium, France, Germany, Israel. A previous version of this paper has been published in the Proceedings of SPIE Volume 10957, Extreme Ultraviolet (EUV) Lithography X. ${ }^{17}$

\section{References}

1. S. Fernandez et al., "Comparative study of extreme ultraviolet absorber materials using lensless actinic imaging," J. Micro/Nanolithogr. MEMS MOEMS 18(1), 013506 (2019).

2. I. Mochi et al., "Actinic inspection of EUV reticles with arbitrary pattern design," Proc. SPIE 10450, 1045007 (2017).

3. I. Mochi et al., "RESCAN: an actinic lensless microscope for defect inspection of EUV reticles," J. Micro/Nanolithogr. MEMS MOEMS 16, 041003 (2017).

4. E. Gallagher et al., "CNTs in the context of EUV pellicle history," Proc. SPIE 10583, 105831E (2018).

5. M. Y. Timmermans et al., "Free-standing carbon nanotube films for extreme ultraviolet pellicle application," J. Micro/Nanolithogr. MEMS MOEMS 17, 043504 (2018).

6. P. Helfenstein et al., "Beam drift and partial probe coherence effects in EUV reflective-mode coherent diffractive imaging," Opt. Express 26, 12242-12256 (2018).

7. C. M. Kewish et al., "Ptychographic characterization of the wavefield in the focus of reflective hard x-ray optics," Ultramicroscopy 110(4), 325-329 (2010).

8. T. Harada et al., "Mask observation results using a coherent extreme ultraviolet scattering microscope at NewSUBARU," J. Vac. Sci. Technol. B: Microelectron. Nanometer Struct. Process. Meas. Phenom. 27(6), 3203-3207 (2009).

9. I. Mochi et al., "Experimental evaluation of the impact of EUV pellicles on reticle imaging," Proc. SPIE 10810, 108100Y (2018).

10. V. Philipsen et al., "Reducing extreme ultraviolet mask three-dimensional effects by alternative metal absorbers," J. Micro/Nanolithogr. MEMS MOEMS 16(4), 041002 (2017). 
11. I. Mochi et al., "Actinic imaging of native and programmed defects on a full-field mask," Proc. SPIE 7636, 76361A (2010).

12. D. Stearns, P. Mirkarimi, and E. Spiller, "Localized defects in multilayer coatings," Thin Solid Films 446(1), 37-49 (2004).

13. W. M. Tong and R. S. Williams, "Kinetics of surface growth: phenomenology, scaling, and mechanisms of smoothening and roughening," Annu. Rev. Phys. Chem. 45(1), 401-438 (1994).

14. M. Mebonia et al., "Experimental studies of the propagation of programmed buried defects into multilayer coatings," in VUV and EUV Metrol., PTB Workshop, vol. 304, 2017, https:// www.euv2017.ptb.de/fileadmin/documents/euv2017/O20_mebonia.pdf.

15. I. Mochi, "Multilayer-mirror," 2019, https://zenodo.org/badge/latestdoi/207020995.

16. S. Bajt et al., "Improved reflectance and stability of Mo/Si multilayers," Opt. Eng. 41(8), 1797-1804 (2002).

17. I. Mochi et al., "Absorber and phase defect inspection on EUV reticles using RESCAN," Proc. SPIE 10957, 109570W (2019).

Iacopo Mochi is an optical physicist. He started working on EUV mask inspection in 2008 at the Center for X-Ray Optics, where he contributed to the design and development of the SHARP microscope. Later, he joined IMEC as an R\&D engineer, studying SRAF solutions to mitigate EUV mask three-dimensional effects. In 2016, he joined Paul Scherrer Institut, where he is currently working on the development of RESCAN, an actinic pattern inspection platform.

Sara Fernandez received her $\mathrm{PhD}$ from the University of Marseille in 2016, in which she used coherent diffraction imaging to study the physical properties of nanostructures under in-situ conditions. Later, she contributed to the RESCAN project through the implementation of CDI for EUV photomasks inspection. Then, she joined the Interactions, Dynamics and Lasers Laboratory, France, to work on a compact high-harmonic generation source for imaging applications.

Ricarda Nebling joined as a PhD candidate in the Paul Scherrer Institut in 2018, where she is working on the development of RESCAN, an actinic patterned mask inspection platform for EUV lithography. Within the RESCAN project, her focus lies on the image reconstruction procedure using phase retrieval algorithms. She has received her bachelor's degree in materials science and her master's degree in micro- and nanosystems from the Swiss Federal Institute of Technologies in Zurich.

Uldis Locans received his PhD from the University of Latvia in 2017, working on the use of new processor architectures for particle accelerator modeling. He joined Paul Scherrer Institut as a scientific software engineer for the RESCAN project in 2018, focusing on high-performance computing needs for ptychography image reconstruction.

Rajendran Rajeev graduated from Tata Institute of Fundamental Research, India, in 2012, where he performed experiments aimed at studying the interaction of intense light pulses with matter. After a year as a guest researcher at the University of Maryland, working on laser-based electron acceleration, he went to ETH, Switzerland, where he was involved into the generation of coherent EUV radiation using laser-based high-harmonic sources. Currently, he is a postdoctoral scientist at Paul Scherrer Institut and is involved with the RESCAN project.

Atoosa Dejkameh is a PhD student at Paul Scherrer Institut. She works on the development of RESCAN, an actinic mask inspection tool at the Swiss light source. She works on experimental and algorithmic methods aiming to improve the resolution of coherent diffraction imaging. During her master's studies at Friedrich-Alexander University, she worked on performance assessment of Fourier ptychography for high NA imaging at Fraunhofer Institute IISB.

Dimitrios Kazazis received his PhD from Brown University, USA, in 2009, working on GeOI tunneling FETs. He did his postdoc until 2014 at CNRS-LPN near Paris, on suspended 2DEGs and quantum Hall effect metrology on graphene. He then joined the Paris Observatory developing THz detectors. Since 2016, he has been working at the Paul Scherrer Institut, Switzerland, 
on advanced lithography and nanofabrication. He has taught several courses at Brown and at Paris 7 University.

Li-Ting Tseng received her $\mathrm{PhD}$ in materials science and engineering from the University of New South Wales, Australia, in 2016, focusing on studying the growth and magnetic properties of diluted magnetic oxide nanostructures and thin films. She currently works as a postdoctoral researcher at Paul Scherrer Institut, Switzerland, on nanofabrication process development using e-beam lithography and EUV interference lithography.

Serhiy Danylyuk is a team leader for EUV and DUV Technology at Fraunhofer Institute for Laser Technology, Germany. He received his diploma in physics from Volyn State University, Ukraine, in 1999. In 2005, he received his $\mathrm{PhD}$ in semiconductor physics from RWTH Aachen University, Germany. Between 2005 and 2008, he was a postdoctoral researcher at Research Center Juelich, Germany. Between 2008 and 2019, he was with RWTH Aachen University, developing applications of EUV radiation for nanopatterning, microscopy, and thin-film characterization. He is an author and coauthor of more than 90 scientific publications in the fields of semiconductor physics and applications of short-wavelength radiation.

Larissa Juschkin received her diploma degree in plasma physics from Novosibirsk State University, Russia, in 1995, and her PhD from Ruhr-University Bochum, Germany, in 2001. In 2012, she was called to a professorship for experimental physics of extreme ultraviolet at RWTH Aachen University. Her scientific interests and activities are concentrated on plasmabased short-wavelength radiation sources and their applications, spectroscopy of highly ionized plasmas, EUV metrology and systems, EUV microscopy and lithography.

Yasin Ekinci received his $\mathrm{PhD}$ from Max Planck Institute for Dynamics and Self Organization in Göttingen, Germany, in 2003. Since 2009, he has been a senior scientist at Paul Scherrer Institut. He is the head of the Advanced Lithography and Metrology Group in Laboratory for Micro- and Nanotechnology. He works on EUV interference lithography and lensless imaging along with other topics in nanoscience. He is a fellow of SPIE. 